\title{
On a Method of Asymptotic Evaluation of Multiple Integrals*
}

\author{
By R. Wong and J. P. McClure
}

\begin{abstract}
In this paper, some of the formal arguments given by Jones and Kline [J. Math. Phys., v. 37, 1958, pp. 1-28] are made rigorous. In particular, the reduction procedure of a multiple oscillatory integral to a one-dimensional Fourier transform is justified, and a Taylor-type theorem with remainder is proved for the Dirac $\delta$-function. The analyticity condition of Jones and Kline is now replaced by infinite differentiability. Connections with the asymptotic expansions of Jeanquartier and Malgrange are also discussed.
\end{abstract}

1. Introduction. In [12] Jones and Kline have given an ingenious derivation of the asymptotic expansions of the double integral

$$
I(\lambda)=\iint_{D} g(x, y) e^{i \lambda f(x, y)} d x d y,
$$

where $D$ is a bounded domain, $g$ and $f$ are real-valued analytic functions in $D$, and $\lambda$ is a large real parameter. Although ingenious, some of their arguments seem to be only formal and require justification. We are particularly concerned with (i) the procedure which they have used to reduce $I(\lambda)$ to a one-dimensional Fourier transform, and (ii) the validity of a Taylor series expansion for the Dirac $\delta$-function. Our objective here is to show that the reduction procedure of Jones and Kline can be made rigorous, and to present a Taylor-type theorem with remainder for the $\delta$-function. In our analysis, $f$ and $g$ need be only sufficiently smooth.

There are several other methods of obtaining asymptotic expansions of $I(\lambda)$. The better known ones, in addition to that of Jones and Kline [12], are due to Focke [6], Chako [3], and Bleistein and Handelsman [1]. The advantages of the Jones-Kline method are: (i) it is easier to calculate the coefficients in various asymptotic expansions of $I(\lambda)$; (ii) it enables one to use the well-developed asymptotic theory for the one-dimensional Fourier integral, including, probably, the error analysis recently established by Olver [14]; and, most importantly, (iii) it gives explicit asymptotic expansions for the Dirac distribution $\delta\{t-f(x, y)\}$ concentrated on the curve $t=f(x, y)$ for small $t$. Asymptotic expansions for such curve (or, more generally, surface) distributions have been obtained by Gel'fand and Shapiro [7], and also by Jeanquartier [10]. In Section 5, we shall give a more detailed discussion in connection with their work.

Received February 4, 1981.

1980 Mathematics Subject Classification. Primary 41A60.

Key words and phrases. Asymptotic expansion, multi-dimensional stationary-phase approximation, Dirac $\delta$-function, surface distribution.

* This research was partially supported by the Natural Sciences and Engineering Research Council of Canada under Grants A7359 and A8069. 
2. Reduction to a Single Integral. In order to reveal the subtlety of the problem in hand, let us briefly repeat some of the arguments given in [12].

Let $m$ and $M$ denote the infimum and supremum of $f(x, y)$ in $D$, respectively. Then

$$
e^{i \lambda f}=\int_{m}^{M} e^{i \lambda t} \delta(t-f) d t
$$

where $\delta$ is the one-dimensional Dirac delta function. Inserting (2.1) in (1.1), we have

$$
I(\lambda)=\iint_{D} g(x, y) \int_{m}^{M} e^{i \lambda t} \delta\{t-f(x, y)\} d t d x d y .
$$

Interchanging the order of integration gives

$$
I(\lambda)=\int_{m}^{M} e^{i \lambda t} h(t) d t
$$

where

$$
h(t)=\iint_{D} g(x, y) \delta\{t-f(x, y)\} d x d y .
$$

The double integral is thus reduced to a single Fourier integral.

Observe that the right-hand side of Eq. (2.1) is not really an integral. It is only a symbolic notation customarily used to represent the Fourier transform of the distribution $\delta(t-f)$. Thus, Eq. (2.1) holds only in the sense of distributions. With this in mind, the interchange of order of integration in (2.2) becomes meaningless. Also, observe that the right side of (2.4) cannot be a double integral, since the set in which the distribution $\delta\{t-f(x, y)\}$ does not vanish has (2-dimensional Lebesgue) measure zero.

One of the ways to legitimately reduce the double integral $I(\lambda)$ to a single Fourier integral is to use the method of resolution of double integrals given in [4, pp. 298-300]. That is, to calculate $I(\lambda)$ by using a subdivision determined by the curves $f(x, y)=$ constant and their orthogonal trajectories. Thus, with $f(x, y)=t$ and $\sigma$ being the arc length of this curve, we have

$$
I(\lambda)=\int_{m}^{M} e^{i \lambda t} h(t) d t
$$

where

$$
h(t)=\int_{f(x, y)=t} \frac{g(x, y)}{\sqrt{f_{x}^{2}+f_{y}^{2}}} d \sigma .
$$

Comparing (2.3)-(2.4) and (2.5)-(2.6) suggests that the correct interpretation of the double "integral" in (2.4) is to view it as the line integral in (2.6). In fact, it is this line integral which is frequently used to define the curve distribution $\delta\{P(x, y)\}$, where $P(x, y)$ is any sufficiently smooth function with $\nabla P=$ $(\partial P / \partial x, \partial P / \partial y)$ nowhere zero on $P(x, y)=0$. Thus, if $g(x, y)$ is a $C^{\infty}$-function with compact support, then we define

$$
\langle\delta(P), g\rangle=\int_{P=0} \frac{g(x, y)}{|\nabla P|} d \sigma ;
$$

see $[8$, p. 222$]$ and $[11$, p. 263$]$. 
There is an alternative definition of the distribution $\delta(P)$, which may serve as a justification for the interchange of the order of integration in (2.2)-(2.3). Let $\theta(P)$ denote the characteristic function of the region $P(x, y)>0$ :

$$
\theta(P)= \begin{cases}0 & \text { for } P<0 \\ 1 & \text { for } P>0\end{cases}
$$

and define the distribution associated with this function by

$$
\langle\theta(P), g\rangle=\iint_{P>0} g(x, y) d x d y .
$$

Since the one-dimensional $\delta$-function is the (distributional) derivative of the Heaviside step function, it is natural to define

$$
\begin{aligned}
\langle\delta(P), g\rangle & =\lim _{c \rightarrow 0} \frac{1}{c}\langle\theta(P+c)-\theta(P), g\rangle \\
& =\lim _{c \rightarrow 0} \frac{1}{c} \iint_{-c<P<0} g(x, y) d x d y .
\end{aligned}
$$

This definition is due to Seeley [16]. By the argument immediately preceding (2.5)-(2.6), it is easily seen that the double integral in (2.10) is equal to

$$
\int_{-c}^{0} \int_{P(x, y)=\xi} \frac{g(x, y)}{|\nabla P|} d \sigma d \xi
$$

see, again, [4, pp. 298-300]. Thus, the above two definitions of $\delta(P)$ agree. Observe that, if we interpret the double "integral" in (2.4) in the sense of the limit in (2.10) with $P(x, y)=t-f(x, y)$, then substituting (2.4) in (2.3) and reversing the order of integration shows that the double integral in (1.1) and the single Fourier integral in (2.3) are indeed equal.

3. A Taylor-Type Expansion for the $\delta$-Function. Let us now return to (1.1) and assume that $(0,0)$ is a critical point of $f$, i.e., $(\nabla f)(0,0)=(0,0)$. For simplicity of illustration, we further assume that $g$ vanishes $C^{\infty}$-smoothly on the boundary of $D$, i.e., $g \in C^{\infty}(D)$ and $g$ together with all its derivatives vanish on $\partial D$, and that the origin is the only critical point of $f(x, y)$ in $D$, i.e., $(\nabla f) \neq(0,0)$ in $D \sim(0,0)$. Under these conditions it is well known that the major contribution to the asymptotic expansion of $I(\lambda)$ comes from the immediate vicinity of the critical point $(0,0)$; see [1, Section 2] and [3, Section 3(a)]. (This fact seems to be particularly transparent from the representation of $I(\lambda)$ given in (2.5)-(2.6).) Thus, without loss of generality, we may suppose that $D$ is some sufficiently small neighborhood of $(0,0)$. The above ideal situation can be realized by using an appropriate van der Corput's neutralizer to isolate the critical point at the origin; see [1] and [3].

As in [12, p. 5], we now set

$$
f(x, y)=f_{0}(x, y)+f_{1}(x, y)
$$

and introduce the new variables

$$
\xi=f_{0}(x, y), \quad \eta=\Psi(x, y)
$$


where $\Psi$ is at our disposal. If we write

$$
F(\xi, \eta)=f_{1}(x, y), \quad \Phi(\xi, \eta)=g(x, y) \frac{\partial(x, y)}{\partial(\xi, \eta)}
$$

and extend $g$ to be identically zero outside $D$, then

$$
\iint_{\mathbf{R}^{2}} g \delta(t-f) d x d y=\iint_{\mathbf{R}^{2}} \Phi \delta(t-\xi-F) d \xi d \eta .
$$

(Here we have used the assumption that $g$ vanishes $C^{\infty}$-smoothly on $\partial D$.) Since both sides of (3.4) are not really double integrals as we have pointed out earlier, this relation holds only formally. The correct interpretation of (3.4) is

$$
\langle\delta(t-f), g\rangle=\langle\delta(t-\xi-F), \Phi\rangle
$$

or, equivalently,

$$
\int_{t=f} \frac{g(x, y)}{|\nabla f|} d \sigma=\int_{t=\xi+F} \frac{\Phi(\xi, \eta)}{|\nabla(\xi+F)|} d \sigma^{\prime},
$$

where $d \sigma^{\prime}$ denotes the length of the curve $t=\xi+F(\xi, \eta)$. In (3.6), the gradient on the left-hand side is taken with respect to the variables $x$ and $y$, whereas the gradient on the right-hand side is taken with respect to $\xi$ and $\eta$. The last identity can be proved by using (2.10) and the familiar change-of-variables formula.

For $k=1,2, \ldots$, we define the derivatives $\delta^{(k)}(P)$ of the distribution $\delta(P)$ by

$$
\delta^{(k)}(P)=\lim _{c \rightarrow 0} \frac{1}{c}\left[\delta^{(k-1)}(P+c)-\delta^{(k-1)}(P)\right] .
$$

It is easy to see that

$$
\left\langle\delta^{(k)}(t-\xi), \varphi\right\rangle=\int_{-\infty}^{\infty} \frac{\partial^{k}}{\partial t^{k}} \varphi(t, \eta) d \eta
$$

for any $C^{\infty}$-function $\varphi(\xi, \eta)$ with compact support.

The idea of Jones and Kline is to expand the $\delta$-function on the right side of (3.5) into a Taylor series, that is, to write

$$
\langle\delta(t-\xi-F), \Phi\rangle=\sum_{r=0}^{\infty} \frac{(-1)^{r}}{r !}\left\langle\delta^{(r)}(t-\xi) F^{r}(\xi, \eta), \Phi\right\rangle .
$$

Such an identity can be easily shown to be true if $F$ is independent of $\xi$, and $\Phi$ is analytic. But, if $F$ depends on $\xi$, the validity of (3.9) requires justification. In what follows, we shall show that under our $C^{\infty}$ - (instead of analyticity) conditions, the $\delta$-function in (3.9) has a finite Taylor expansion plus an explicit remainder term, from which the remaining analysis of Jones and Kline can be continued and the various asymptotic expansions of $I(\lambda)$ be derived.

We first need the following lemma, a proof of which can be found in the appendix.

LEMMA 1. Let $f$ and $g$ be $C^{\infty}$-functions, and let $n$ be a nonnegative integer. Then

$$
\begin{aligned}
{\left[f(x) g^{n+1}(x)\right]^{(n+1)}=} & -\sum_{p=0}^{n}\left(\begin{array}{l}
n+1 \\
p+1
\end{array}\right)\left[f(x) g^{n-p}(x)\right]^{(n+1)}[-g(x)]^{p+1} \\
& +(n+1) ! f(x)\left[g^{\prime}(x)\right]^{n+1}
\end{aligned}
$$


LEMMA 2. Let $\Phi(\xi, \eta)$ be a $C^{\infty}$-function with compact support, and $F(\xi, \eta)$ be a $C^{\infty}$-function with $\partial F / \partial \xi>-1$ on the support of $\Phi$. If $\xi_{t}$ denotes the solution $\xi_{t}(\eta)$ to the equation $t=\xi+F(\xi, \eta)$, then for any $n \geqslant 0$

$$
\langle\delta(t-\xi-F), \Phi\rangle=\sum_{r=0}^{n} \frac{(-1)^{r}}{r !}\left\langle F^{r}(\xi, \eta) \delta^{(r)}(t-\xi), \Phi\right\rangle+R_{n+1}(t),
$$

where

$$
R_{n+1}(t)=\int_{-\infty}^{\infty} r_{n+1}(t, \eta) d \eta
$$

and

$$
\begin{aligned}
r_{n+1}(t, \eta)= & \frac{\Phi\left(\xi_{t}, \eta\right)\left[-F_{\xi}\left(\xi_{t}, \eta\right)\right]^{n+1}}{1+F_{\xi}\left(\xi_{t}, \eta\right)} \\
& +\sum_{r=0}^{n} \frac{1}{r !(n-r) !} \int_{t}^{\xi_{t}}(t-\mu)^{r} \frac{\partial^{n+1}}{\partial \mu^{n+1}}\left\{\Phi(\mu, \eta)[-F(\mu, \eta)]^{n-r}\right\} d \mu
\end{aligned}
$$

Proof. Since $1+F_{\xi}>0$, by the implicit function theorem, there exists a solution $\xi_{t}(\eta)$ to the equation $t=\xi+F(\xi, \eta)$, and hence we may write explicitly

$$
d \sigma^{\prime}=\frac{\sqrt{\left[1+F_{\xi}\left(\xi_{t}, \eta\right)\right]^{2}+\left[F_{\eta}\left(\xi_{t}, \eta\right)\right]^{2}}}{1+F_{\xi}\left(\xi_{t}, \eta\right)} d \eta
$$

From this and the line integral on the right-hand side of (3.6), it follows that

$$
\langle\delta(t-\xi-F), \Phi\rangle=\int_{-\infty}^{\infty} \frac{\Phi\left(\xi_{t}, \eta\right)}{1+F_{\xi}\left(\xi_{t}, \eta\right)} d \eta
$$

Since distributions multiplied by $C^{\infty}$-functions are, by definition, the distributions acting on test functions multiplied by the $C^{\infty}$-functions, we also have from (3.8)

$$
\left\langle\delta^{(r)}(t-\xi) F^{r}(\xi, \eta), \Phi\right\rangle=\int_{-\infty}^{\infty} \frac{\partial^{r}}{\partial t^{r}}\left[\Phi(t, \eta) F^{r}(t, \eta)\right] d \eta .
$$

Thus, $r_{n+1}(t, \eta)$, as given in (3.12), satisfies

$$
r_{n+1}(t, \eta)=\frac{\Phi\left(\xi_{t}, \eta\right)}{1+F_{\xi}\left(\xi_{t}, \eta\right)}-\sum_{r=0}^{n} \frac{(-1)^{r}}{r !} \frac{\partial^{r}}{\partial t^{r}}\left[\Phi(t, \eta) F^{r}(t, \eta)\right] .
$$

To show that (3.13) holds, we proceed by induction. When $n=0$, the result follows immediately from the fundamental theorem of calculus. Assuming that the statement is true for $n=k$, one can show that it holds also for $n=k+1$ by (i) inserting (3.13) (with $n=k$ ) in

$$
r_{k+2}(t, \eta)=r_{k+1}(t, \eta)-\frac{(-1)^{k+1}}{(k+1) !} \frac{\partial^{k+1}}{\partial t^{k+1}}\left[\Phi(t, \eta) F^{k+1}(t, \eta)\right],
$$

(ii) integrating the terms under the sum by parts, and (iii) adding and subtracting the terms

$$
\Phi\left(\xi_{t}, \eta\right)\left[-F_{\xi}\left(\xi_{t}, \eta\right)\right]^{k+1}
$$


and

$$
\frac{(-1)^{k+1}}{(k+1) !}\left[\frac{\partial^{k+1}}{\partial \xi^{k+1}}\left\{\Phi(\xi, \eta) F^{k+1}(\xi, \eta)\right\}\right]_{\xi=\xi} .
$$

Here use is made also of the identity in Lemma 1. This completes the proof of Lemma 2.

4. The Behavior of $h(t)$ Near the Origin. From the theory of one-dimensional Fourier integrals, it is now well known that the asymptotic behavior of $I(\lambda)$, given in (2.5), as $\lambda \rightarrow \infty$, is completely determined by the behavior of the function $h(t)$, given in (2.6), in the neighborhood of $t=0$. In this section we shall show that under our $C^{\infty}$ (instead of analyticity) conditions set in Sections 2 and 3, the function $h(t)$ indeed possesses asymptotic expansions near $t=0$. Since much of our analysis is similar to that given in [12, Section 5], we shall present only the case in which $(0,0)$ is a local extremum of $f(x, y)$.

Since $f$ is a $C^{\infty}$-function, we may expand $f(x, y)$ into a finite Taylor series with remainder. The linear terms do not appear, because $(0,0)$ is a critical point. The constant term can be omitted because it contributes only the factor $\exp \{i \lambda f(0,0)\}$ outside the integral. Hence,

$$
f(x, y)=\left\{f_{20} x^{2}+f_{11} x y+f_{02} y^{2}\right\}+\text { higher terms. }
$$

By a simple rotation of coordinates, we may, without loss of generality, assume that the cross-product $x y$ vanishes, i.e., $f_{11}=0$; see [2, p. 326]. This simplification is made, essentially, in all derivations of the multi-dimensional stationary-phase approximation. Thus, the first few nonzero terms of $f$ are given by

$$
f(x, y)=f_{20} x^{2}+f_{02} y^{2}+\text { cubic terms }+\ldots
$$

Let us assume that both $f_{20}$ and $f_{02}$ are positive, i.e., $(0,0)$ is a local minimum. Put

$$
x=\xi^{1 / 2} \frac{\cos \eta}{f_{2 \sigma^{2}}^{1 / 2}}, \quad y=\xi^{1 / 2} \frac{\sin \eta}{f_{02}^{1 / 2}} .
$$

Then

$$
\xi=f_{20} x^{2}+f_{02} y^{2}
$$

and

$$
\frac{\partial(x, y)}{\partial(\xi, \eta)}=\frac{1}{\sqrt{4 f_{20} f_{02}}}
$$

Since $f(x, y)=\xi+F(\xi, \eta)$ by (3.1)-(3.3), we have, from (4.1) and (4.2), $1+\partial F / \partial \xi$ $=\partial f / \partial \xi>0$ on the support of $\Phi$. (Keep in mind that the support of $\Phi$ can be taken to be any shape and arbitrarily small as long as it contains an open disk with center at the origin; see the first paragraph of Section 3.) Since $(0,0)$ is a local minimum, $m=0$ in (2.5) and $h(t)=0$ for $t<0$ in (2.6). Recall that

$$
h(t)=\langle\delta(t-f), g\rangle=\langle\delta(t-\xi-F), \Phi\rangle .
$$

Combining (3.11), (3.15) and (4.2), we have for $t>0$

$$
h(t)=\sum_{r=0}^{n} \frac{\partial^{r}}{\partial t^{r}} \int_{0}^{2 \pi} \frac{(-1)^{r}}{r !}\left[\Phi(t, \eta) F^{r}(t, \eta)\right] d \eta+R_{n+1}(t)
$$


Since $f$ and $g$ are $C^{\infty}$, so are $\Phi$ and $F$. Therefore, by (4.2), for any $N>1$,

$$
\frac{(-1)^{r}}{r !} \Phi(\xi, \eta) F^{r}(\xi, \eta)=\sum_{\mu=0}^{N-1} c_{\mu}(\eta) \xi^{\mu / 2}+O\left(\xi^{N / 2}\right)
$$

where $c_{\mu}(\eta)$ is a polynomial in $\cos \eta$ and $\sin \eta$, and the $O$-symbol is independent of $\eta$. Since it is easily shown that

$$
\int_{0}^{2 \pi} c_{\mu}(\eta) d \eta=0, \quad \mu=1,3,5, \ldots,
$$

(cf. lines 7 and 8 in [12, p. 16]), each term in the series of (4.6) has an asymptotic expansion, as $t \rightarrow 0^{+}$, of the form $\sum_{\nu=0}^{\infty} a_{v r} t^{\nu}$, where the coefficients $a_{v r}$ are constants. If we can show that

$$
R_{n+1}(t)=O\left(t^{(n+1) / 2}\right), \quad \text { as } t \rightarrow 0^{+},
$$

then we succeed in establishing the asymptotic expansion

$$
h(t) \sim \sum_{\nu=0}^{\infty} a_{\nu} t^{\nu}, \quad \text { as } t \rightarrow 0^{+} .
$$

The coefficients $a_{\nu}$ are of course those already given in [12, Eq. (33)].

Here we wish to point out that we have not established the equality in (33) of [12] (even under the stronger assumption of analyticity). What we have shown is that Eq. (33) there holds at least asymptotically. This is, nevertheless, all one needs to derive the desired asymptotic expansion of the double integral $I(\lambda)$.

To prove (4.8), we return to (3.12) and (3.13). Note that, as $\xi \rightarrow 0^{+}$, we have

$$
F(\xi, \eta) \sim \sum_{\nu=3}^{\infty} b_{\nu}(\eta) \xi^{\nu / 2}
$$

where $b_{\nu}(\eta)$ is a polynomial in $\cos \eta$ and $\sin \eta$. This series can be termwise differentiated to yield an asymptotic series for $F_{\xi}(\xi, \eta)$. By substituting (4.10) into the equation $t=\xi+F(\xi, \eta)$ and inverting the resulting series, we have

$$
\xi_{t} \sim t+\sum_{\nu=3}^{\infty} d_{\nu}(\eta) t^{\nu / 2}, \quad \text { as } t \rightarrow 0
$$

The first term on the right side of (3.13) is now clearly of the order $O\left(t^{(n+1) / 2}\right)$. Each of the integrals under the summation sign in (3.13) is also of this order, since $\left|\xi_{t}-t\right|=O\left(t^{3 / 2}\right)$, and hence

$$
\int_{t}^{\xi_{1}}(t-\mu)^{r} \mu^{(n-3 r-2) / 2} d \mu=O\left(t^{(n+1) / 2}\right)
$$

as $t \rightarrow 0^{+}$. This proves that $r_{n+1}(t, \eta)=O\left(t^{(n+1) / 2}\right)$ as $t \rightarrow 0^{+}$. Since the 0 -symbol here is independent of $\eta$, the validity of (4.8) is therefore established.

Here we wish to point out that if we assume that $f$ and $g$ in (4.5) have only a finite number of continuous derivatives, then a finite (instead of an infinite) asymptotic expansion of the form (4.9) can still be obtained for $h(t)$ and hence for the double integral $I(\lambda)$ in (1.1). Also, it is obvious that this weaker assumption will not cause any complication in the derivation of the final result.

When $f_{20}$ and $f_{02}$ are both negative, i.e., $(0,0)$ is a local maximum, we have $M=0$ in (2.5) and $h(t)=0$ for $t>0$ in (2.6). The analysis in this case remains essentially unchanged. 
To conclude this section, we also make a remark concerning the change of variables

$$
\xi=f_{20} x^{2}+f_{02} y^{2} \text { and } \eta=x
$$

made by Jones and Kline, in the case when $f_{20}>0$ and $f_{02}<0$, i.e., $(0,0)$ is a saddle point, see $[12$, p. 16 , Section 5.2]. Since $\partial(\xi, \eta) / \partial(x, y)=0$ when $y=0$, the Jacobian $\partial(x, y) / \partial(\xi, \eta)$ in (3.3) does not exist there, although their result is still correct since the integral after the transformation converges as an improper Riemann integral. However, it appears to us that it is more natural to let

$$
x=\xi^{1 / 2} \frac{\cosh \eta}{f_{20}^{1 / 2}}, y=\xi^{1 / 2} \frac{\sinh \eta}{\left(-f_{02}\right)^{1 / 2}} .
$$

Then we have

$$
\xi=f_{20} x^{2}+f_{02} y^{2}
$$

as in (4.3), and the Jacobian in this case is a constant

$$
\frac{\partial(x, y)}{\partial(\xi, \eta)}=\frac{1}{\sqrt{-4 f_{20} f_{02}}}
$$

Using our transformation (4.13), we need consider integrals of the form

$$
I_{m, n}^{*}=\int_{\eta_{1}(t)}^{\eta_{2}(t)} \cosh ^{m} \eta \sinh ^{n} \eta d \nu
$$

where $m+n$ is a nonnegative even integer and

$$
\eta_{1}(t)=0, \quad \eta_{2}(t)=\cosh ^{-1}\left[d\left(f_{20} / t\right)^{1 / 2}\right] \quad(d>0) .
$$

These integrals can be easily evaluated with the aid of integral tables, and they are related to those $I_{m, n}$ considered by Jones and Kline. In fact, a simple change of variable shows

$$
I_{m, n}=\frac{t^{m+n}}{f_{20}^{m+1 / 2}} I_{m, n}^{*}
$$

5. Remarks on Asymptotic Expansions of Surface Distributions. From the analysis in [12] and the present note, it is evident that the method of Jones and Kline can be applied to integrals of higher dimensions. In a more recent paper [13], Malgrange has also studied the asymptotic behavior of the integral

$$
I(\lambda)=\int_{R^{n}} g(x) e^{i \lambda f(x)} d x,
$$

as $\lambda \rightarrow+\infty$, where $f$ is a real analytic function on $R^{n}$ and $g$ is a $C^{\infty}$-function on $R^{n}$ with compact support. More specifically, he has shown that this oscillatory integral has an asymptotic expansion of the form

$$
I(\lambda) \sim \sum c_{\alpha, q} \lambda^{-\alpha-1}(\log \lambda)^{q},
$$

where $0<q \leqslant n-1$ and $\alpha$ runs through a countable set of positive rational numbers; see [13, Eq. (7.4)]. Although Malgrange has pointed out that the $\alpha$ 's and $q$ 's are related to the Picard-Lefschetz monodromy of $f$ at 0 , no explicit expressions are given for these exponents; see, also, [9] and [5, p. 262]. This is mainly because 
of a result due to Jeanquartier, which Malgrange has employed in the derivation of (5.2). Malgrange's approach can essentially be described as follows. Proceeding as in Section 2 of the present note, he first reduces the multiple integral (5.1) to a one-dimensional Fourier transform by writing

$$
I(\lambda)=\int_{-\infty}^{\infty} e^{i \lambda t}\langle\delta(f-t), g\rangle d t
$$

where $\langle\delta(f-t), g\rangle$ is the surface distribution defined by (2.7); see [13, Eqs. (6.1) and (7.1)], and then termwise integrates the asymptotic expansions

$$
\langle\delta(f-t), g\rangle \sim \sum a_{\alpha, q}^{( \pm)}|t|^{\alpha}(\log |t|)^{q}
$$

as $t \rightarrow 0^{ \pm}$; see [13, Section 7]. The existence of these expansions has been established earlier by Jeanquartier [10], who has also shown that these expansions can be differentiated term by term to give asymptotic expansions of the successive derivatives of $\langle\delta(f-t), g\rangle$. However, the method of Jeanquartier leads to only an existence result, whereas that of Jones and Kline can be used to construct asymptotic expansions of the form (5.4) in most cases. Furthermore, Jeanquartier (and hence Malgrange) assumes that $f$ is real analytic, whereas the result of Jones and Kline now only requires $f$ to be $C^{\infty}$ (in view of Lemma 2 of the present note). In fact, it is these differences that have motivated us to make the formal arguments in [12] rigorous. Another point which we wish to make is that, when the coefficients $a_{\alpha, q}^{( \pm)}$in (5.4) are explicitly known, the asymptotic expansion (5.4) may become simpler. For instance, in the case when $n=2$ and the origin is a saddle point, the asymptotic expansion of $\langle\delta(f-t), g\rangle$ is given by

$$
\langle\delta(f-t), g\rangle \sim \sum c_{s}^{( \pm)} t^{s} \log |t|, \quad \text { as }|t| \rightarrow 0^{+},
$$

where the coefficients $c_{s}^{( \pm)}$satisfy $c_{s}^{(+)}=c_{s}^{(-)}$for all $s \geqslant 0$; see [12, Eq. (37)]. Therefore the asymptotic expansion of $I(\lambda)$ in this case is of the form

$$
I(\lambda) \sim \sum_{s=0}^{\infty} a_{s} \lambda^{-s-1}, \quad \text { as } \lambda \rightarrow+\infty,
$$

and does not involve logarithms; see [12, the paragraph immediately following Eq. (37)].

To conclude this paper, we also call attention to an asymptotic expansion, similar to (5.4), given in Gel'fand and Shilov [8, p. 326]. Let $f$ and $g$ be $C^{\infty}$ functions on $R^{n}$ and $g$ have compact support. Assume that the hypersurface $f=0$ consists only of reducible points (see $[8$, p. 313]) and that the hypersurfaces $f=t$ with $t>0$ have no singular points at all (i.e., $(\nabla f)(x) \neq 0$ for all $x$ on $f=t$ ). Then it has been shown that

$$
\langle\delta(f-t), g\rangle \sim \sum_{k=1}^{\infty} \sum_{m=1}^{m_{k}} a_{k, m} t^{\lambda_{k}-1}(\log t)^{m-1}, \quad \text { as } t \rightarrow 0^{+},
$$

where the $\lambda_{k}$ 's are positive rational numbers arranged in an increasing order and the $m_{k}$ 's are nonnegative integers. This result is actually due to Gel'fand and 
Shapiro [7], and their method proceeds as follows. First they show that the function $F(\lambda)$, defined by

$$
F(\lambda)=\int_{f>0} f^{\lambda}(x) g(x) d x, \quad\left(x \in R^{n}\right),
$$

is meromorphic in $\lambda$ and that its poles can be arranged in a decreasing order

$$
-\lambda_{1},-\lambda_{2}, \ldots, \lambda_{k}, \ldots ; \quad 0<\lambda_{1}<\lambda_{2}<\ldots<\lambda_{k}<\ldots
$$

Next they observe that putting

$$
I(t)=\langle\delta(f-t), g\rangle
$$

gives

$$
F(\lambda)=\int_{0}^{\infty} I(t) t^{\lambda} d t
$$

(This identity can be proved by using the method of resolution of multiple integrals mentioned in Section 2.) The integral on the right-hand side is the Mellin transform of $I(t)$ evaluated at $\lambda+1$. Thus, by the Mellin inversion formula,

$$
I(t)=\frac{1}{2 \pi i} \int_{c-i \infty}^{c+i \infty} t^{-\lambda-1} F(\lambda) d \lambda .
$$

Finally, shifting the contour of integration to the left, they obtain the desired expansion (5.7). The terms in the series (5.7) are thus picked up as residues, the integer $m_{k}$ is the multiplicity of the pole at $-\lambda_{k}$, and $a_{k, m}$ is $(-1)^{m} /(m-1)$ ! times the coefficient of $\left(\lambda+\lambda_{k}\right)^{-m}$ in the Laurent expansion of $F(\lambda)$ about the pole at $-\lambda_{k}$.

Although the result of Gel'fand and Shapiro given in (5.7) appears to be more explicit than that of Jeanquartier given in (5.4), the determination of the locations of the poles of $F(\lambda)$ and the calculation of the coefficients $a_{k, m}$ in (5.7) are still difficult tasks.

It is obvious that the asymptotic expansions mentioned in this section are intimately related to the work of Jones and Kline, this fact, however, does not seem to have been observed before.

Appendix. In this appendix we shall prove Lemma 1 . The case $n=0$ is essentially the product rule. For $n \geqslant 1$, we need the following identity [15, Section 0.43 , p. 19], which is known as the Leibniz rule for composite functions: For any positive integer $r$,

$$
(f \circ g)^{(r)}=\sum_{j=1}^{r} f^{(i)} \circ g \sum_{\alpha} c_{\alpha, j, r} \prod_{i=1}^{r}\left(g^{(i)}\right)^{\alpha_{i}}
$$

where the coefficients $c_{\alpha, j, r}$ are positive integers, and the second sum runs over all multi-indices $\alpha=\left(\alpha_{1}, \ldots, \alpha_{r}\right)$ of nonnegative integers such that $\alpha_{1}+\cdots+\alpha_{r}=$ $j$ and $\alpha_{1}+2 \alpha_{2}+\cdots+r \alpha_{r}=r$. First we consider the left-hand side of (3.10). The following steps are straightforward. 
ASYMPTOTIC EVALUATION OF MULTIPLE INTEGRALS

519

$$
\begin{aligned}
& {\left[f \frac{g^{n+1}}{(n+1) !}\right]^{(n+1)}=\sum_{j=0}^{n+1}\left(\begin{array}{c}
n+1 \\
j
\end{array}\right) f^{(n+1-j)}\left[\frac{g^{n+1}}{(n+1) !}\right]^{(j)} } \\
&=f^{(n+1)} \frac{g^{n+1}}{(n+1) !}+\sum_{j=1}^{n}\left(\begin{array}{c}
n+1 \\
j
\end{array}\right) f^{(n+1-j)} \sum_{p=1}^{j} \frac{g^{n+1-p}}{(n+1-p) !} \\
& \times \sum_{\alpha} c_{\alpha, p, j} \prod_{i=1}^{j}\left(g^{(i)}\right)^{\left(\alpha_{i}\right)} \\
&+f \sum_{p=1}^{n} \frac{g^{n+1-p}}{(n+1-p) !} \sum_{\alpha} c_{\alpha, p, n+1} \prod_{i=1}^{n+1}\left(g^{(i)}\right)^{\alpha_{i}}+f\left(g^{\prime}\right)^{n+1} \\
&= f^{(n+1)} \frac{g^{n+1}}{(n+1) !}+f\left(g^{\prime}\right)^{n+1} \\
&+\sum_{p=1}^{n} \sum_{j=p}^{n+1}(n+1) f^{(n+1-j)} \frac{g^{(n+1-p)}}{(n+1-p) !} \sum_{\alpha} c_{\alpha, p, j} \\
& \times \prod_{i=1}^{j}\left(g^{(i)}\right)^{\alpha_{i} .}
\end{aligned}
$$

Next we consider the right-hand side of (3.10). We obtain

$$
\begin{aligned}
&-\sum_{p=0}^{n}\left[f \frac{g^{n-p}}{(n-p) !}\right]^{(n+1)} \frac{(-g)^{p+1}}{(p+1) !}+f\left(g^{\prime}\right)^{n+1} \\
&= f\left(g^{\prime}\right)^{n+1}-\sum_{p=0}^{n} \sum_{j=0}^{n+1}\left(\begin{array}{c}
n+1 \\
j
\end{array}\right) f^{(n+1-j)}\left[\frac{g^{n-p}}{(n-p) !}\right]^{(j)} \frac{(-g)^{p+1}}{(p+1) !} \\
&= f\left(g^{\prime}\right)^{n+1}-\sum_{p=0}^{n} f^{(n+1)} g^{n+1} \frac{(-1)^{p+1}}{(n-p) !(p+1) !} \\
&-\sum_{p=0}^{n-1} \sum_{j=1}^{n-p}\left(\begin{array}{c}
n+1 \\
j
\end{array} f^{(n+1-j)} \sum_{r=1}^{j} \frac{g^{n-p-r}}{(n-p-r) !} \sum_{\alpha} c_{\alpha, r, j}\right. \\
& \times \prod_{i=1}^{j}\left(g^{(i)}\right)^{\alpha_{i}} \frac{(-g)^{p+1}}{(p+1) !} \\
&-\sum_{p=0}^{n-1} \sum_{j=n-p+1}^{n+1}\left(\begin{array}{c}
n+1 \\
j
\end{array}\right) f^{(n+1-j)} \sum_{r=0}^{n-p} \frac{g^{n-p-r}}{(n-p-r) !} \\
& \times \sum_{\alpha} c_{\alpha, r, j} \prod_{i=1}^{j}\left(g^{(i)}\right)^{\alpha_{i}} \frac{(-g)^{p+1}}{(p+1) !} \\
&= f\left(g^{\prime}\right)^{n+1}+f^{(n+1)} \frac{g^{n+1}}{(n+1) !} \\
&-\sum_{p=1}^{n} \sum_{j=1}^{n-p+1}(n+1) f^{(n+1-j)} \sum_{r=1}^{j} \frac{g^{n-p-r+1}}{(n-p-r+1) !} \\
& \times \sum_{\alpha} c_{\alpha, r, j} \prod_{i=1}^{j}\left(g^{(i)}\right)^{\alpha_{i}} \frac{(-g)^{p}}{p !}
\end{aligned}
$$


(Equation (A.2) continued)

$$
\begin{aligned}
& -\sum_{p=1}^{n} \sum_{j=n-p+2}^{n+1}\left(\begin{array}{c}
n+1 \\
j
\end{array}\right) f^{(n+1-j)} \sum_{r=1}^{n-p+1} \frac{g^{n-p-r+1}}{(n-p-r+1) !} \\
& \times \sum_{\alpha} c_{\alpha, r, j} \prod_{i=1}^{j}\left(g^{(i)}\right)^{\alpha_{i}} \frac{(-g)^{p}}{p !} \\
& =f\left(g^{\prime}\right)^{n+1}+f^{(n+1)} \frac{g^{n+1}}{(n+1) !} \\
& -\sum_{p=1}^{n} \sum_{r=1}^{n-p+1} \sum_{j=r}^{n-p+1}\left(\begin{array}{c}
n+1 \\
j
\end{array}\right) f^{(n+1-j)} \frac{g^{n-r+1}(-1)^{p}}{(n-p-r+1) ! p !} \\
& \times \sum_{\alpha} c_{\alpha, r, j} \prod_{i=1}^{j}\left(g^{(i)}\right)^{\alpha_{i}} \\
& -\sum_{p=1}^{n} \sum_{r=1}^{n-p+1} \sum_{j=n-p+2}^{n+1}\left(\begin{array}{c}
n+1 \\
j
\end{array}\right) f^{(n+1-j)} \frac{g^{n-r+1}(-1)^{p}}{(n-p-r+1) ! p !} \\
& \times \sum_{\alpha} c_{\alpha, r, j} \prod_{i=1}^{j}\left(g^{(i)}\right)^{\alpha_{i}} \\
& =f\left(g^{\prime}\right)^{n+1}+f^{(n+1)} \frac{g^{n+1}}{(n+1) !} \\
& -\sum_{r=1}^{n} \sum_{j=r}^{n+1}\left(\begin{array}{c}
n+1 \\
j
\end{array}\right) f^{(n+1-j)}\left(\sum_{p=1}^{n-r+1} \frac{(-1)^{p}}{(n-p-r+1) ! p !}\right) \\
& \times g^{n-r+1} \sum_{\alpha} c_{\alpha, r, j} \prod_{i=1}^{j}\left(g^{(i)}\right)^{\alpha_{i}} \\
& =f\left(g^{\prime}\right)^{n+1}+f^{(n+1)} \frac{g^{n+1}}{(n+1) !} \\
& +\sum_{r=1}^{n} \sum_{j=r}^{n+1}\left(\begin{array}{c}
n+1 \\
j
\end{array}\right) f^{(n+1-j)} \frac{g^{n-r+1}}{(n-r+1) !} \sum_{\alpha} c_{\alpha, r, j} \\
& \times \prod_{i=1}^{j}\left(g^{(i)}\right)^{\alpha_{i}}
\end{aligned}
$$

This is exactly the same as the expression in (A1), thus proving Lemma 1.

Department of Mathematics

University of Manitoba

Winnipeg, Manitoba, Canada R3T 2N2

1. N. Bleistein \& R. A. HANDelsman, "Multidimensional stationary phase. An alternative derivation," SIAM J. Math. Anal., v. 6, 1975, pp. 480-487.

2. N. Bleistein \& R. A. Handelsman, Asymptotic Expansions of Integrals, Holt, Rinehart and Winston, New York, 1975.

3. N. CHAKo, "Asymptotic expansions of double and multiple integrals arising in diffraction theory," J. Inst. Math. Appl., v. 1, 1965, pp. 372-422.

4. R. Courant, Differential and Integral Calculus, vol. 2, Blackie \& Son Ltd., London, 1970.

5. J. J. DuistermaAt, "Oscillatory integrals, Lagrange immersions and unfoldings of singularities," Comm. Pure Appl. Math., v. 27, 1974, pp. 207-281. 
6. J. Focke, “Asymptotische Entwicklungen mittels der Methode der stationären phase,” Ber. Verh. Sächs. Akad. Wiss. Leipzig Math.-Nat. Kl., v. 101, 1954, pp. 1-48.

7. M. Gel'fand \& Z. YA. Shapiro, "Homogeneous functions and their extensions," Amer. Math. Soc. Transl. (2), v. 8, 1958, pp. 21-85.

8. I. M. Gel'fand \& G. E. Shrov, Generalized Functions, vol. 1, Academic Press, New York, 1964.

9. H. A. HAMM, Remarks on Asymptotic Integrals, the Polynomial of I. N. Berstein and the Picard-Lefschetz Monodromy, Proc. Sympos. Pure Math., vol. 30, Amer. Math. Soc., Providence, R.I., 1977, pp. 31-35.

10. P. Jeanquartier, “Développement asymptotique de la distribution de Dirac," C.R. Acad. Sci. Paris Sér. A, v. 271, 1970, pp. 1159-1161.

11. D. S. Jones, Generalized Functions, McGraw-Hill, London, 1966.

12. D. S. JoNES \& M. KLINE, "Asymptotic expansions of multiple integrals and the method of stationary phase," J. Math. Phys., v. 37, 1958, pp. 1-28.

13. B. Malgrange, "Intégrales asymptotique et monodromie," Ann. Sci. Ecole Norm Sup. (4), v. 7, 1974, pp. 405-430.

14. F. W. J. OlvER, "Error bounds for stationary phase approximations," SIAM J. Math. Anal., v. 5, 1974, pp. 19-29.

15. I. M. Ryshix \& I. S. GradsteIn, Table of Integrals, Series, and Products, Academic Press, New York, 1965.

16. R. T. SeELeY, Distributions on Surfaces, Report T.W. 78, Mathematical Centre, Amsterdam, 1962. 